\title{
LUMINOUS INFRARED GALAXIES WITH THE SUBMILLIMETER ARRAY. III. THE DENSE KILOPARSEC MOLECULAR CONCENTRATIONS OF Arp 299
}

\author{
Kazimierz Sliwa $^{1}$, Christine D. Wilson ${ }^{1}$, Glen R. PetitPas ${ }^{2}$, Lee Armus ${ }^{3}$, Mika Juvela ${ }^{4}$, \\ Satoki Matsushita ${ }^{5}$, Alison B. PeCK ${ }^{6}$, and Min S. Yun ${ }^{7}$ \\ ${ }^{1}$ Department of Physics and Astronomy, McMaster University, Hamilton, ON L8S 4M1 Canada; sliwak@mcmaster.ca, wilson@physics.mcmaster.ca \\ ${ }^{2}$ Harvard-Smithsonian Center for Astrophysics, Cambridge, MA 02138, USA; gpetitpa@cfa.harvard.edu \\ ${ }^{3}$ Spitzer Science Center, California Institute of Technology, Pasadena, CA 91125, USA; lee@ipac.caltech.edu \\ ${ }^{4}$ University of Helsinki Observatory, Finland; mika.juvela@ helsinki.fi \\ ${ }^{5}$ Academia Sinica Institute of Astronomy and Astrophysics, Taipei 106, Taiwan; satoki@asiaa.sinica.edu.tw \\ ${ }^{6}$ Joint ALMA Office, Avda El Golf 40, piso 18, Santiago, Chile 7550108; apeck@alma.cl \\ ${ }^{7}$ Department of Astronomy, University of Massachusetts, Amherst, MA 01003, USA; myun@astro.umass.edu \\ Received 2011 October 11; accepted 2012 April 28; published 2012 June 13
}

\begin{abstract}
We have used high-resolution $\left(\sim 2\right.$ '. 3 ) observations of the local ( $D_{\mathrm{L}}=46 \mathrm{Mpc}$ ) luminous infrared galaxy Arp 299 to map out the physical properties of the molecular gas that provides the fuel for its extreme star formation activity. The ${ }^{12} \mathrm{CO} J=3-2,{ }^{12} \mathrm{CO} J=2-1$, and ${ }^{13} \mathrm{CO} J=2-1$ lines were observed with the Submillimeter Array, and the short spacings of the ${ }^{12} \mathrm{CO} J=2-1$ and $J=3-2$ observations have been recovered using the James Clerk Maxwell Telescope single dish observations. We use the radiative transfer code RADEX to estimate the physical properties (density, column density, and temperature) of the different regions in this system. The RADEX solutions of the two galaxy nuclei, IC 694 and NGC 3690, are consistent with a wide range of gas components, from warm moderately dense gas with $T_{\text {kin }}>30 \mathrm{~K}$ and $n\left(\mathrm{H}_{2}\right) \sim 0.3-3 \times 10^{3} \mathrm{~cm}^{-3}$ to cold dense gas with $T_{\text {kin }} \sim 10-30 \mathrm{~K}$ and $n\left(\mathrm{H}_{2}\right)>3 \times 10^{3} \mathrm{~cm}^{-3}$. The overlap region is shown to have a better constrained solution with $T_{\text {kin }} \sim 10-50 \mathrm{~K}$ and $n\left(\mathrm{H}_{2}\right) \sim 1-30 \times 10^{3} \mathrm{~cm}^{-3}$. We estimate the gas masses and star formation rates of each region in order to derive molecular gas depletion times. The depletion times of all regions (20-50 Myr) are found to be about two orders of magnitude lower than those of normal spiral galaxies. This rapid depletion time can probably be explained by a high fraction of dense gas on kiloparsec scales in Arp 299. We estimate the CO-to- $\mathrm{H}_{2}$ factor, $\alpha_{\text {co }}$ to be $0.4 \pm 0.3\left(3 \times 10^{-4} / x_{\mathrm{CO}}\right) M_{\odot}\left(\mathrm{K} \mathrm{km} \mathrm{s}^{-1} \mathrm{pc}^{2}\right)^{-1}$ for the overlap region. This value agrees well with values determined previously for more advanced merger systems.
\end{abstract}

Key words: galaxies: individual (Arp 299) - galaxies: interactions - galaxies: starburst - submillimeter: galaxies

Online-only material: color figures

\section{INTRODUCTION}

Ultra/Luminous infrared galaxies (U/LIRGs) are systems in the local universe that exhibit extreme star formation. They emit a large portion of their total luminosity at farinfrared (FIR) wavelengths (LIRGs: $L_{\mathrm{FIR}} \sim 10^{11}-10^{12} L_{\odot}$; ULIRGs: $L_{\mathrm{FIR}}>10^{12} L_{\odot}$; Sanders \& Mirabel 1996). Early optical studies have shown that a large fraction of U/LIRGs show morphologies that resemble systems that are interacting or merging (Armus et al. 1987; Sanders et al. 1988). High-resolution optical and near-infrared imaging has revealed that a large fraction of U/LIRGs have nuclear separations of 0.3-48 kpc (Murphy et al. 1996). The merger process likely triggers the extreme star formation in these galaxies (Sanders et al. 1988) but the detailed process is still not well understood.

U/LIRGs have been observed to contain a large amount of molecular gas with $M\left(\mathrm{H}_{2}\right)$ up to about $10^{10} M_{\odot}$ (Sanders et al. 1986). The molecular gas is seen to be concentrated near the nuclear regions within a radius of $0.5 \mathrm{kpc}$ (Sanders \& Mirabel 1996). Subarcsecond resolution CO observations have revealed rotating disks of molecular gas that has been driven to the nuclei (Downes \& Solomon 1998). Theoretical models have shown that during the collision, the gas loses angular momentum due to dynamical friction which causes the gas to decouple from the stars and flow inward toward the nuclei (Barnes \& Hernquist 1991). The concentrated molecular gas provides fuel for star formation and/or for an active galactic nucleus (AGN).
Menéndez-Delmestre et al. (2009) have shown that submillimeter galaxies (SMGs) have similar radiation environments to local starbursts. The distribution of the optical depth determined from the silicate absorption feature at $9.7 \mu \mathrm{m}$ was found to be lower than for local U/LIRGs, which suggests that the mid-infrared regions of SMGs have a lower dust obscuration. The difference in extinction between U/LIRGs and SMGs likely arises from differences in geometry. Using ${ }^{12} \mathrm{CO} J=3-2 \mathrm{ob}-$ servations, Iono et al. (2009) have shown that the molecular gas disks in local U/LIRGs are more compact $(0.3-3.1 \mathrm{kpc})$ than the SMGs (3-16 kpc). Menéndez-Delmestre et al. (2009) also found that the majority of SMGs are dominated by intense star formation just like local U/LIRGs. The differences suggest that ULIRGs are not exact local analogs of high-redshift SMGs; however, there are enough similarities that local ULIRGs are often used as templates for SMGs.

Arp 299 (NGC 3690 + IC 694, Mrk 171, VV 118, IRAS $11257+5850)$ is one of the nearest $\left(D_{\mathrm{L}}=46 \mathrm{Mpc}\right)$ examples of a merger system. It has a far-infrared luminosity of $L_{\mathrm{FIR}}=$ $5.5 \times 10^{11} L_{\odot}$ (Sanders et al., 2003), classifying it as an LIRG. In the optical, the two nuclei are still distinguishable. Highresolution observations show the nuclei are separated by $22^{\prime \prime}$ (4.9 kpc ; Sargent \& Scoville 1991). Gehrz et al. (1983) showed that there is also a third region of emission thought to be associated with the overlap of the two galaxies. Hibbard \& Yun (1999) found two $180 \mathrm{kpc} \mathrm{HI}$ tails as well as a faint optical tail displaced from the Hi tails. They infer that the 
merger started 750 Myr ago and the two nuclei will completely merge in roughly 60 Myr. Evolutionary starburst models have shown that Arp 299 has been going through a recent episode of interaction-induced star formation over a span of about $15 \mathrm{Myr}$ (Alonso-Herrero et al. 2000). Interferometric observations of ${ }^{12} \mathrm{CO}$ show strong emission from the three major regions found in infrared images (Sargent \& Scoville 1991; Aalto et al., 1997; Casoli et al., 1999) indicating the presence of large amounts of molecular gas.

Studying the physical conditions and distribution of the warm, dense gas associated with star formation will help in understanding the processes and timescales controlling the star formation in galaxy mergers. In this paper, we analyze Submillimeter Array (SMA) interferometric observations of Arp 299 in the ${ }^{12} \mathrm{CO} J=2-1,{ }^{13} \mathrm{CO} J=2-1$ and ${ }^{12} \mathrm{CO} J=3-2$ lines first published in Wilson et al. (2008, hereafter Paper I). In Section 2, we present the observations and the data reduction process including the short spacings correction. In Section 3, we present and discuss our radiative transfer modeling results as well the $\mathrm{CO}$-to- $\mathrm{H}_{2}$ conversion factor, $\alpha_{\mathrm{co}}$ for the extranuclear region. In Section 4, we discuss the star formation rates and depletion times for each region of Arp 299.

\section{OBSERVATIONS AND DATA REDUCTION}

\subsection{Submillimeter Array}

We have used the SMA (Ho et al. 2004) observations centered on ${ }^{12} \mathrm{CO} J=2-1$ and ${ }^{12} \mathrm{CO} J=3-2$ for Arp 299 first published in Paper I. The correlator was configured to have a spectral resolution of $0.8125 \mathrm{MHz}$ corresponding to roughly $1.1 \mathrm{~km} \mathrm{~s}^{-1}$ for ${ }^{12} \mathrm{CO} J=2-1$ and $0.7 \mathrm{~km} \mathrm{~s}^{-1}$ for ${ }^{12} \mathrm{CO} J=3-2$. The ${ }^{12} \mathrm{CO} J=2-1$ transition was centered in the upper sideband and ${ }^{13} \mathrm{CO} J=2-1$ was observed simultaneously in the lower sideband $10 \mathrm{GHz}$ away. Since Arp 299 has sufficiently extended emission, a small mosaic of two pointings was used. Further details of the observations are given in Paper I.

We start with the calibrated $u v$ data from Paper I. These data were converted to FITS files and then exported into CASA format for further processing and imaging. The data were flagged to remove the first six and last six channels of each of the 24 chunks of the correlator. The data were also flagged to remove high amplitude value data points (removing less than $1 \%$ of the data). The $u v$ data sets were continuum subtracted using line-free channels. Clean data cubes with velocity resolutions of $20 \mathrm{~km} \mathrm{~s}^{-1}$ and $50 \mathrm{~km} \mathrm{~s}^{-1}$ were created using a robust weighting scheme in order to achieve a good balance between resolution and sensitivity. Each data set was cleaned down to two times the rms noise in each velocity channel (Table 1). The beam was sufficiently clean and the emission is sufficiently compact that cleaning the entire inner quarter region produces good maps. However, for ${ }^{12} \mathrm{CO} J=2-1$ and $J=3-2$, we interactively placed clean boxes around emission regions in each channel. Integrated intensity maps were created with a $2 \sigma$ cutoff using velocity channels with emission from Arp 299 (Figure 1). Finally, the integrated intensity maps were corrected for the primary beam.

\subsection{Owens Valley Radio Observatory}

We have obtained a clean data cube of ${ }^{12} \mathrm{CO} J=1-0$ observed using the Owens Valley Radio Observatory (OVRO) from S. Aalto first published in Aalto et al. (1997). Details of the observations and reduction are given in Aalto et al. (1997). An integrated intensity map was created with a $2 \sigma$ cutoff using velocity channels with emission from Arp 299 (see
Table 1

Interferometric Data for Arp 299

\begin{tabular}{lccc}
\hline \hline CO Transition Line & $\begin{array}{c}\text { Total Flux } \\
\left(\text { Jy km s }^{-1}\right)\end{array}$ & $\begin{array}{c}\text { Beam } \\
\left({ }^{\prime \prime}\right)\end{array}$ & $\begin{array}{c}\text { rms }^{\mathrm{b}} \\
\left(\mathrm{mJy} \mathrm{beam}^{-1}\right)\end{array}$ \\
\hline${ }^{12} \mathrm{CO} J=1-0^{\mathrm{c}}$ & $377 \pm 9$ & $2.5 \times 2.2$ & 9 \\
${ }^{12} \mathrm{CO} J=2-1$ & $1870 \pm 30$ & $3.0 \times 1.8$ & 17 \\
${ }^{13} \mathrm{CO} J=2-1$ & $40 \pm 2$ & $3.2 \times 1.8$ & 10 \\
${ }^{12} \mathrm{CO} J=3-2$ & $2820 \pm 40$ & $2.2 \times 1.9$ & 33
\end{tabular}

Notes.

${ }^{a}$ Measurement uncertainty only; calibration uncertainty is $20 \%$ (Paper I).

${ }^{\mathrm{b}} \mathrm{rms}$ values for a $20 \mathrm{~km} \mathrm{~s}^{-1}$ channel width for all lines except ${ }^{13} \mathrm{CO} J=2-1$ which uses $50 \mathrm{~km} \mathrm{~s}^{-1}$ channel width.

c Map obtained from Aalto et al. (1997); observed with Owens Valley Radio Observatory.

Figure 1). The integrated intensity map was corrected for the primary beam. Adding this line to our analysis will help to better constrain the density and temperature of Arp 299.

\subsection{James Clerk Maxwell Telescope}

Arp 299 was observed with the James Clerk Maxwell Telescope (JCMT) on 2007 March 31 for ${ }^{12} \mathrm{CO} J=3-2$ (Program: M07AC11; PI: C.D. Wilson) and 2011 March 9 for ${ }^{12} \mathrm{CO} J=$ 2-1 (Program: M11AC06; PI: K. Sliwa). The JCMT was used to make a $2^{\prime}$ map with $6^{\prime \prime}$ sampling using the array receiver HARP-B in the ${ }^{12} \mathrm{CO} J=3-2$ line with a $15^{\prime \prime}$ beam and to make a $7^{\prime \prime}$ sampled $1.5 \times 1^{\prime}$ map with the RxA receiver centered on the ${ }^{12} \mathrm{CO} J=2-1$ line with a $22^{\prime \prime}$ beam. Further details of the ${ }^{12} \mathrm{CO} J=3-2$ data are given in Paper I. The raw data were made into a cube that spanned from $-400 \mathrm{~km} \mathrm{~s}^{-1}$ to $400 \mathrm{~km} \mathrm{~s}^{-1}$ using the Starlink software (Currie et al. 2008) written for JCMT data. The cube was then baseline corrected using line-free channels and a first-order baseline. Several of the outer bad pixels were trimmed off and the cube was binned to a velocity resolution of $20 \mathrm{~km} \mathrm{~s}^{-1}$.

We assume a main beam efficiency $\left(\eta_{\mathrm{mb}}\right)$ of 0.6 for ${ }^{12} \mathrm{CO} \mathrm{J}=$ 3-2 and 0.69 for ${ }^{12} \mathrm{CO} J=2-1$ in order to convert the intrinsic units of antenna temperature $\left(T_{\mathrm{A}}^{*}\right)$ to main beam temperature $\left(T_{\mathrm{mb}}=T_{\mathrm{A}}^{*} / \eta_{\mathrm{mb}}\right)$ and then to $\mathrm{Jy} \mathrm{km} \mathrm{s}^{-1}$ which gives us a scaling factor of $26.9 \mathrm{Jy} \mathrm{K}\left(T_{\mathrm{mb}}\right)^{-1}$ and $22.9 \mathrm{Jy} \mathrm{K}\left(T_{\mathrm{mb}}\right)^{-1}$ for ${ }^{12} \mathrm{CO}$ $J=3-2$ and ${ }^{12} \mathrm{CO} J=2-1$, respectively. We thus assume that emission from Arp 299 fills the beam, unlike Paper I where Arp 299 was treated like a point source.

\subsection{Short Spacing Correction}

We use the JCMT maps to recover the short spacings of the SMA maps. We combine the two maps in the image plane using the feather task in CASA. Both maps were binned to the same velocity resolution so that each velocity channel matches in each map. The feathering technique takes both maps, regrids the lowresolution image to match the high-resolution image, Fourier transforms them to the gridded $u v$-plane, sums up the gridded visibilities and then Fourier transforms the feathered map back into the image plane (Figure 2). Comparing the SMA only maps with the JCMT maps, the ${ }^{12} \mathrm{CO} J=2-1$ map was missing about $20 \%$ of the total flux and the ${ }^{12} \mathrm{CO} J=3-2$ map was missing about $70 \%$ of the total flux (Table 2). Note that Paper I estimated only $47 \%$ missing flux in ${ }^{12} \mathrm{CO} J=3-2$ because they assumed Arp 299 is point-like in the $\mathrm{K}$ to Jy conversion of the single dish data. Casoli et al. (1999) have compared the OVRO ${ }^{12} \mathrm{CO} J=$ 1-0 map of Aalto et al. (1997) with an IRAM 30 m single dish 

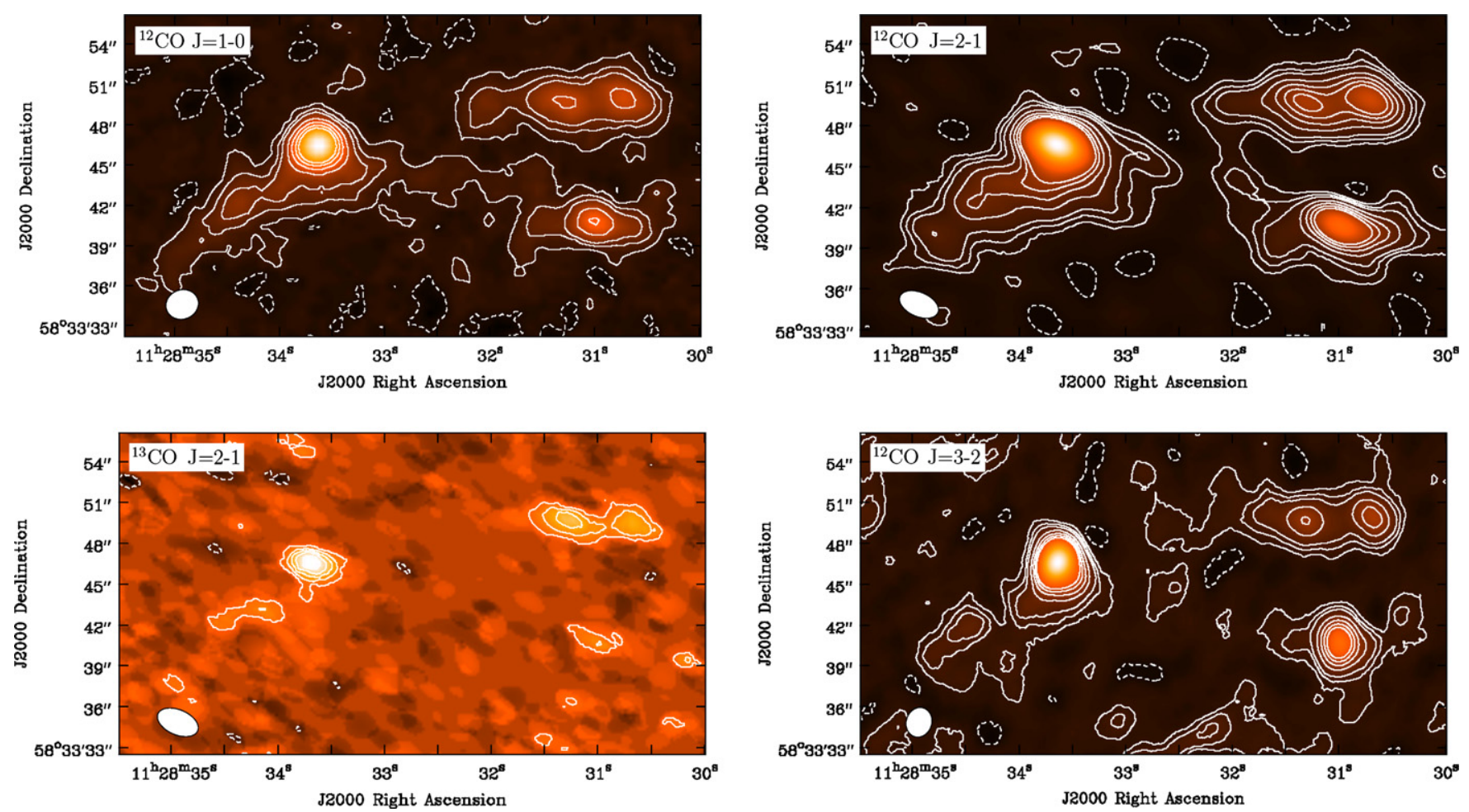

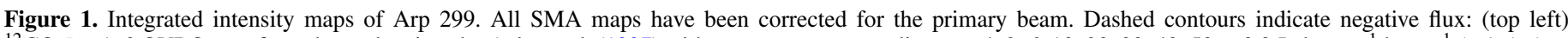

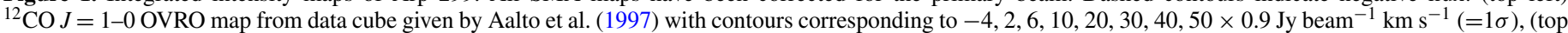

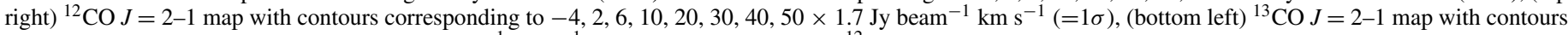

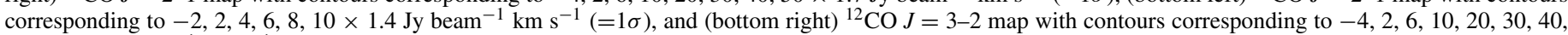
$50 \times 3.5 \mathrm{Jy} \mathrm{beam}^{-1} \mathrm{~km} \mathrm{~s}^{-1}(=1 \sigma)$. Note: the SMA maps from Paper I have been reprocessed using CASA.

(A color version of this figure is available in the online journal.)

Table 2

Comparison of JCMT and Combined (Feathered) Maps ${ }^{\mathrm{a}}$

\begin{tabular}{|c|c|c|c|c|c|}
\hline \multirow[b]{2}{*}{$\begin{array}{l}\text { Transition } \\
\left({ }^{12} \mathrm{CO}\right)\end{array}$} & \multicolumn{3}{|c|}{ Total Flux $\left(\mathrm{Jy} \mathrm{km} \mathrm{s}^{-1}\right)^{\mathrm{b}}$} & \multirow[b]{2}{*}{$\begin{array}{c}\text { Missing }^{\mathrm{c}} \\
(\%)\end{array}$} & \multirow[b]{2}{*}{$\begin{array}{c}\text { Recovered }^{\mathrm{d}} \\
(\%)\end{array}$} \\
\hline & SMA & Feather & JCMT & & \\
\hline$J=2-1$ & $1870 \pm 30$ & $2380 \pm 30$ & $2320 \pm 120$ & $20 \pm 30$ & $100 \pm 30$ \\
\hline$J=3-2$ & $2820 \pm 40$ & $6350 \pm 50$ & $8770 \pm 120$ & $70 \pm 30$ & $70 \pm 40$ \\
\hline
\end{tabular}

Notes.

${ }^{13} \mathrm{CO} J=2-1$ and ${ }^{12} \mathrm{CO} J=1-0$ single dish maps are not available.

b Measurement uncertainty only; calibration uncertainty is $20 \%$ for SMA and the JCMT ${ }^{12} \mathrm{CO} J=2-1$ maps and $30 \%$ for the JCMT ${ }^{12} \mathrm{CO} J=3-2$ (Paper I). ${ }^{c}$ Missing flux in the SMA only map relative to the JCMT map. Uncertainty calculation includes calibration uncertainty.

${ }^{\mathrm{d}}$ Recovered flux in the feathered map relative to the JCMT map. Uncertainty calculation includes calibration uncertainty.

map and found that about $56 \%$ of the flux was missing. The missing flux in all the maps is an issue but we try to avoid the problem by using the peak integrated intensities for our analysis (see Section 3.1).

\subsection{Line Ratio Maps}

For our analysis, we degraded the resolution of all the interferometric maps to 3". $6 \times 2$ ". 4 in order to probe the molecular gas on similar physical scales. The elongated beam of the $\mathrm{CO}$ $J=2-1$ maps limited the best possible degraded resolution to 3 ." $6 \times 2$ 2. 4 . We applied a Gaussian taper weighting to the SMA $u v$ data to degrade the resolution. The resolution of the OVRO ${ }^{12} \mathrm{CO} J=1-0$ map was degraded by applying a Gaussian kernel to the data cube. New integrated intensity maps were made from these resolution matched cubes. We converted the intrinsic units of the CO integrated intensity maps from Jy beam ${ }^{-1} \mathrm{~km} \mathrm{~s}^{-1}$ to $\mathrm{K} \mathrm{km} \mathrm{s}^{-1}$. We then created ${ }^{12} \mathrm{CO}((J=3-2) /(J=2-1)),{ }^{12} \mathrm{CO}$ $((J=2-1) /(J=1-0))$ and $\left({ }^{12} \mathrm{CO} /{ }^{13} \mathrm{CO}\right) J=2-1$ line ratio maps by dividing the appropriate maps with a cutoff at $2 \sigma$ for each map (Figure 3 ). Note that the line ratio values near the edges of each region are noisier because the uncertainty by definition approaches $\pm 50 \%$.

\section{RADIATIVE TRANSFER MODELING}

\subsection{Physical Conditions in Arp 299}

We adopt the notation of Gehrz et al. (1983) to denote each region of Arp 299. The two nuclei are denoted as region A for IC 694 and region B for NGC 3690. We denote the "disk" of region $\mathrm{A}$ as region $\mathrm{A} 2$. The strong emission to the north of region $\mathrm{B}$, denoted as region $\mathrm{C}$, can be broken up into two sub regions; the strongest peak to the west is denoted as $\mathrm{C} 1$ and the peak to the east is denoted as C2 (Figure 2). Note that our sub-region $\mathrm{C} 2$ is the region $\mathrm{C} 2+\mathrm{C} 3$ in Aalto et al. (1997).

The line width and size of the $\mathrm{CO}$ emission of each region have been measured using the ${ }^{12} \mathrm{CO} J=3-2$ map which has the best angular resolution. The velocity line width FWHM of each region was fitted with a Gaussian and the results are presented in Table 3. The diameter of each region was obtained by fitting to a two-dimensional Gaussian. For region $\mathrm{C}$, each sub-region, $\mathrm{C} 1$ and $\mathrm{C} 2$, was fitted to a two-dimensional Gaussian because the entire region cannot be fitted well by a single Gaussian. The source sizes listed in Table 3 are deconvolved from the intrinsic ${ }^{12} \mathrm{CO} J=3-2$ beam size. 

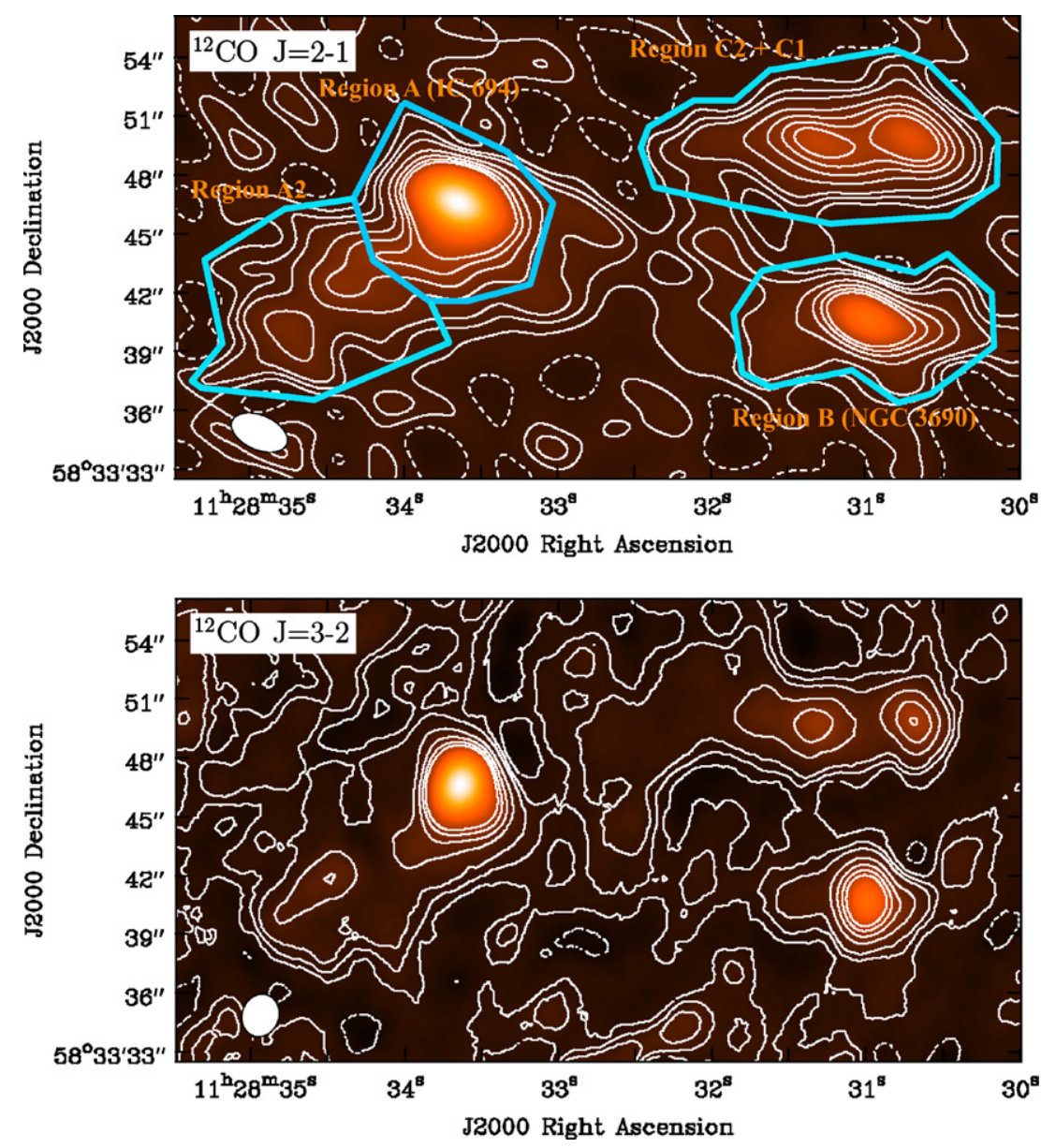

Figure 2. Feathered (short spacing corrected) SMA maps: (top) ${ }^{12} \mathrm{CO} J=2-1$ map with contour levels as in Figure 1 . The light blue lines indicate the area where the flux was integrated for each region (see Table 6); (bottom) ${ }^{12} \mathrm{CO} J=3-2$ map with contour levels as in Figure 1.

(A color version of this figure is available in the online journal.)

Table 3

Source Size and Dynamical Masses

\begin{tabular}{lcccc}
\hline \hline Region & $\begin{array}{c}\text { Velocity FWHM } \\
\left(\mathrm{km} \mathrm{s}^{-1}\right)\end{array}$ & \multicolumn{2}{c}{ Deconvolved Source Diameter } & \\
\cline { 3 - 4 } & 325 & $(1.7 \pm 0.5) \times(1.2 \pm 1.0)$ & $380 \times 270$ & $(3.4 \pm 2.1)$ \\
\hline A (IC 694) & 90 & $\ldots$ & $\ldots$ & $\ldots$ \\
A2 (IC 694 disk) & 185 & $(2.0 \pm 0.6) \times(1.4 \pm 1.0)$ & $450 \times 310$ & $(1.3 \pm 0.7)$ \\
B (NGC 3690) & 80 & $(2.5 \pm 0.8) \times(1.6 \pm 1.3)$ & $560 \times 360$ & $(0.3 \pm 0.2)$ \\
C1 (Overlap) & 80 & $(4.3 \pm 2.1) \times(1.5 \pm 1.4)$ & $960 \times 330$ & $(0.4 \pm 0.3)$ \\
C2 (Overlap) & & & \\
\hline
\end{tabular}

Notes.

${ }^{\text {a }}$ Uncertainty in velocity FWHM is $10 \mathrm{~km} \mathrm{~s}^{-1}$.

b Beam size is 2 ." $2 \times 1$ 1.9.

${ }^{\mathrm{c}}$ Region A2 cannot be fit with a Gaussian because it is too close to region A.

Using the line ratio maps, we obtain the line ratio values at the peak intensity pixel of each region found in the ${ }^{12} \mathrm{CO} J=$ 3-2 map. The line ratios and $1 \sigma$ values are presented in Table 4 . The $1 \sigma$ values are calculated assuming a $20 \%$ calibration uncertainty for each map for the ${ }^{12} \mathrm{CO}((J=3-2) /(J=2-1))$ and ${ }^{12} \mathrm{CO}((J=2-1) /(J=1-0))$ line ratios and a $10 \%$ calibration uncertainty for each map for the $\left({ }^{12} \mathrm{CO} /{ }^{13} \mathrm{CO}\right) J=2-1$ line ratio since these maps were observed simultaneously. We see that including short spacings with the JCMT maps does not change the peak ratio values significantly except for region A2. We assume that the missing flux for ${ }^{12} \mathrm{CO} J=1-0$ and ${ }^{13} \mathrm{CO} J=$
$2-1$ at the peak intensity value is insignificant for regions $\mathrm{A}, \mathrm{B}$, $\mathrm{C} 1$ and $\mathrm{C} 2$. The ${ }^{12} \mathrm{CO}((J=2-1) /(J=1-0))$ and $\left({ }^{12} \mathrm{CO} /{ }^{13} \mathrm{CO}\right)$ $J=2-1$ line ratios for region A2 is more uncertain which may result in unreliable radiative transfer results.

To constrain the average temperature and density of each region, we use the radiative transfer code RADEX (van der Tak et al. 2007). RADEX assumes a homogeneous medium making it a useful tool to constrain the physical conditions such as density and temperature using observational data. We created a three-dimensional grid of models varying $N\left({ }^{12} \mathrm{CO}\right)$ in steps of $0.1 \times 10^{18} \mathrm{~cm}^{-2}$ from $10^{17}$ to $10^{20} \mathrm{~cm}^{-2}, T_{\text {kin }}$ in steps of 

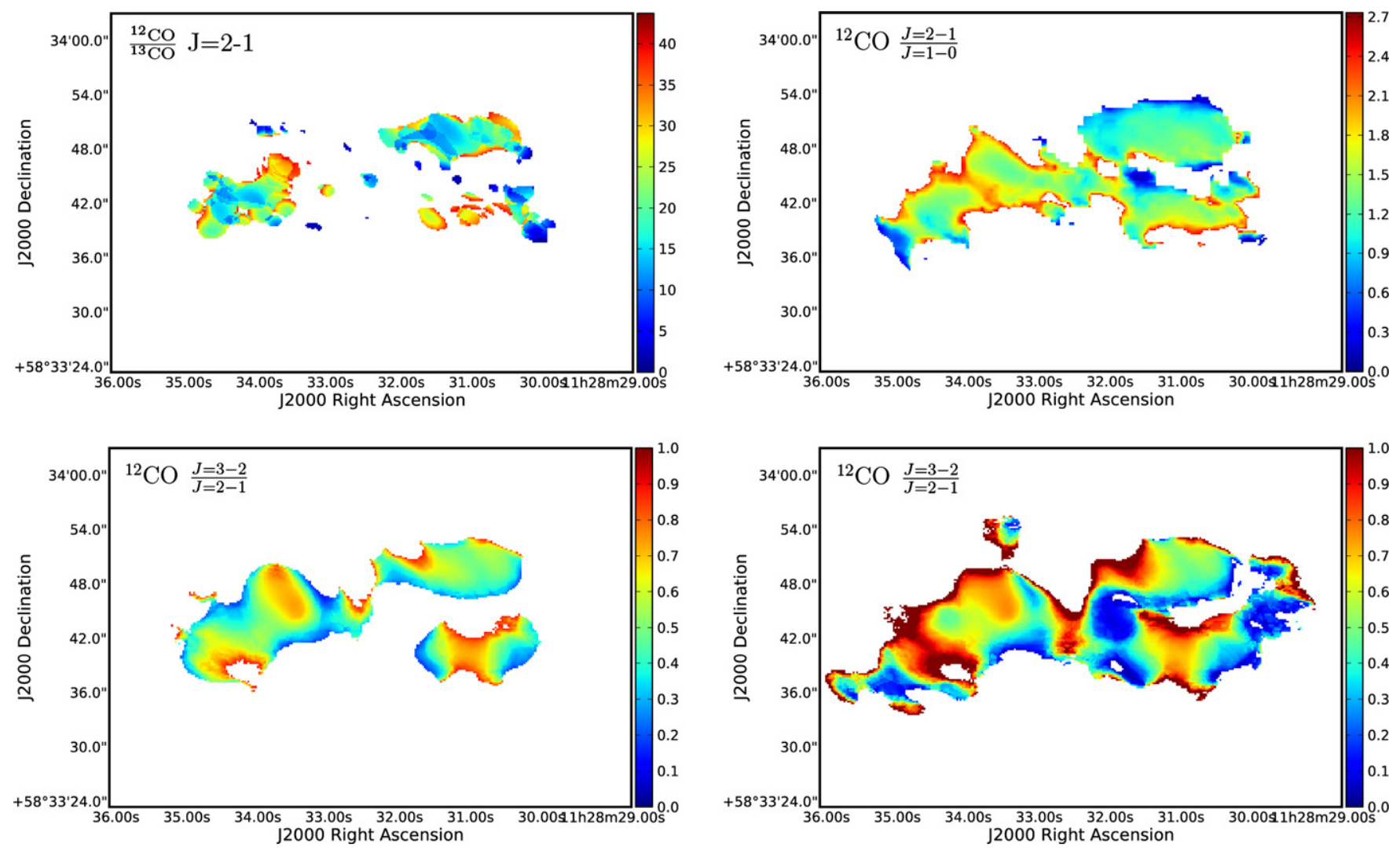

Figure 3. Line ratio maps: (top left) $\left({ }^{12} \mathrm{CO} /{ }^{13} \mathrm{CO}\right) J=2-1$; (top right) ${ }^{12} \mathrm{CO}((J=2-1) /(J=1-0))$; (bottom left) $\mathrm{SMA}$ only ${ }^{12} \mathrm{CO}((J=3-2) /(J=2-1))$; (bottom right) feathered ${ }^{12} \mathrm{CO}((J=3-2) /(J=2-1))$ ratio map. Only emission that is $>2 \sigma$ in both maps is included.

(A color version of this figure is available in the online journal.)

Table 4

CO Line Ratios of Arp 299

\begin{tabular}{lcccc}
\hline \hline Region & ${ }^{12} \mathrm{CO} \frac{J=3-2 \mathrm{~b}}{J=2-1}$ & ${ }^{12} \mathrm{CO} \frac{J=3-2 \mathrm{c}}{J=2-1}$ & ${ }^{12} \mathrm{CO} \frac{J=2-1}{J=1-0}$ & $\frac{{ }^{12} \mathrm{CO}}{{ }^{13} \mathrm{CO}} J=2-1$ \\
\hline $\mathrm{A}$ & $0.74 \pm 0.21$ & $0.72 \pm 0.20$ & $1.37 \pm 0.39$ & $30.9 \pm 4.4$ \\
$\mathrm{~A} 2$ & $0.76 \pm 0.22$ & $0.58 \pm 0.16$ & $1.32 \pm 0.38$ & $12.6 \pm 1.8$ \\
$\mathrm{~B}$ & $0.73 \pm 0.21$ & $0.73 \pm 0.21$ & $1.66 \pm 0.47$ & $27.3 \pm 3.9$ \\
$\mathrm{C} 1$ & $0.52 \pm 0.15$ & $0.51 \pm 0.15$ & $1.24 \pm 0.35$ & $17.2 \pm 2.4$ \\
$\mathrm{C} 2$ & $0.65 \pm 0.19$ & $0.60 \pm 0.17$ & $1.23 \pm 0.35$ & $11.6 \pm 1.6$ \\
\hline
\end{tabular}

Notes.

${ }^{a}$ All ratios are in terms of integrated brightness temperatures at the peak intensity value found in the ${ }^{12} \mathrm{CO} J=3-2$ map. Uncertainties include calibration errors only. All maps were degraded to a resolution of 3". $6 \times 2$ '. 4 .

${ }^{\mathrm{b}}$ Using short spacing corrected maps.

${ }^{c}$ Using SMA only maps.

$1 \mathrm{~K}$ from 10 to $1000 \mathrm{~K}$, and $\log \left(n\left(\mathrm{H}_{2}\right)\right)$ in steps of 0.02 from 2 to 8 . We adopt a ${ }^{12} \mathrm{CO}$ to ${ }^{13} \mathrm{CO}$ abundance ratio of 50 . We used the line widths $(d V)$ from Table 3 to convert $N\left({ }^{12} \mathrm{CO}\right) / d V$ to $N\left({ }^{12} \mathrm{CO}\right)$. The RADEX solutions were plotted as $T_{\text {kin }}$ versus $n\left(\mathrm{H}_{2}\right)$ with contours for each line ratio $\pm 1 \sigma$. The solutions are picked out where the three line ratios overlap within the $\pm 1 \sigma$ range by inspection by eye (Figure 4). For regions A, A2, and B, a wide range of solutions exist. In particular, there are solutions with low $(<30 \mathrm{~K})$ temperature but density only constrained to be $>10^{4} \mathrm{~cm}^{-3}$ and also solutions with lower densities but temperature only constrained to be $>30 \mathrm{~K}$. The solutions for regions $\mathrm{A}, \mathrm{A} 2$, and $\mathrm{B}$ suggest that the pressure of the medium is $\mathrm{P} / \mathrm{k} \gtrsim 10^{5} \mathrm{~K} \mathrm{~cm}^{-3}$. Aalto et al. (1997) estimated a temperature of $>50 \mathrm{~K}$ and a density of $10^{4}-10^{5} \mathrm{~cm}^{-3}$ for region A using ${ }^{12} \mathrm{CO}$ and ${ }^{13} \mathrm{CO} J=1-0$ and $\mathrm{HCN} J=1-0$ line ratios. This
Table 5

RADEX Results

\begin{tabular}{lccc}
\hline \hline Region & $\begin{array}{c}N\left({ }^{12} \mathrm{CO}\right) \\
\left(\mathrm{cm}^{-2}\right)\end{array}$ & $\begin{array}{c}T_{\text {kin }} \\
(\mathrm{K})\end{array}$ & $\begin{array}{c}n\left(\mathrm{H}_{2}\right) \\
\left(\mathrm{cm}^{-3}\right)\end{array}$ \\
\hline A & $1-8 \times 10^{18}$ & $10-500$ & $>10^{2.5}$ \\
A2 & $2-30 \times 10^{18}$ & $20-500$ & $>10^{2.5}$ \\
B & $1-6 \times 10^{18}$ & $10-1000$ & $>10^{2.5}$ \\
C1 & $1-4 \times 10^{18}$ & $10-50$ & $10^{3}-10^{4.5}$ \\
C2 & $2-9 \times 10^{18}$ & $10-200$ & $10^{3}-10^{5}$ \\
\hline
\end{tabular}

is not consistent with our RADEX solutions, but our analysis consists of more $\mathrm{CO}$ lines and we do not assume local thermal equilibrium (LTE). The HCN $J=1-0$ line traces molecular gas at a higher critical density than our ${ }^{12} \mathrm{CO} J=3-2$ line; however, the HCN lines may be affected by IR pumping through a $14 \mu \mathrm{m}$ vibrational transition (Aalto et al. 1995) and in extreme IR environments can be overluminous with respect to the lines of other dense molecular gas tracers (Graciá-Carpio et al. 2006). If the $\mathrm{HCN} J=1-0$ line is overluminous, radiative transfer solutions would show higher densities than the true value. For the sub-regions $\mathrm{C} 1$ and $\mathrm{C} 2$, we have better constrained solutions for both temperature and density (Table 5). For sub-region C2, the solutions show a slightly wider temperature, density and column density range than for region $\mathrm{C} 1$. The optical depth of the solutions for sub-regions $\mathrm{C} 1$ and $\mathrm{C} 2$ was found to be $\sim 0.5-1.5$, $\sim 2.5-4$, and $\sim 4$ for ${ }^{12} \mathrm{CO} J=1-0, J=2-1$, and $J=3-2$, respectively. The low optical depth $(<1)$ of ${ }^{12} \mathrm{CO} J=1-0$ line is likely responsible for the unusual $(>1) 2-1 / 1-0$ line ratios seen in Arp 299. The assumption of a homogeneous medium adds 

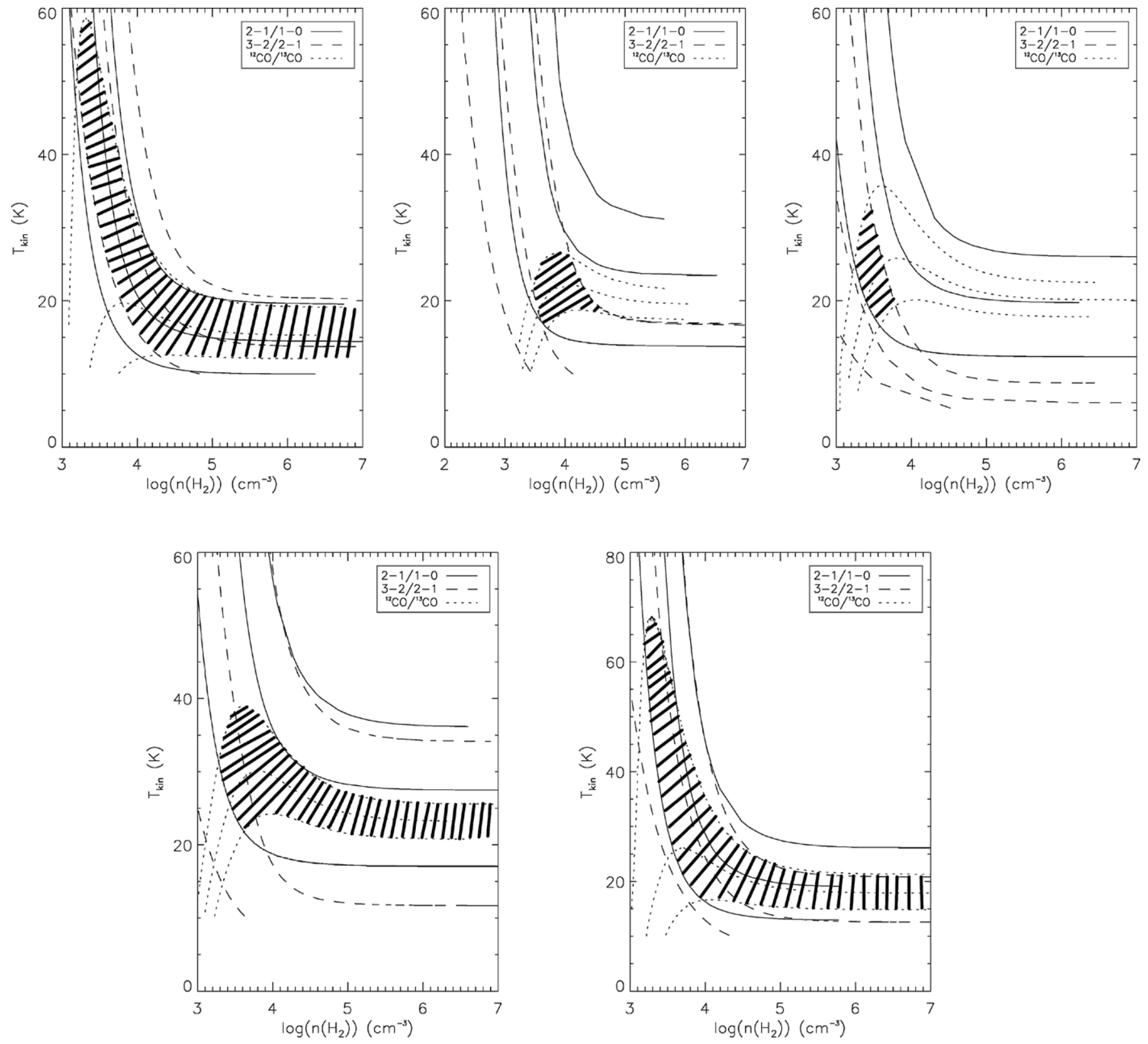

Figure 4. Sample RADEX plots of each region of Arp 299: (top left) Region A for $N\left({ }^{12} \mathrm{CO}\right)=2 \times 10^{18} \mathrm{~cm}^{-2}$, (top middle) Sub-region $\mathrm{C} 2 \mathrm{for} N\left({ }^{12} \mathrm{CO}\right)=3 \times 10^{18} \mathrm{~cm}{ }^{-2}$, (top left) Sub-region $\mathrm{C} 1$ for $N\left({ }^{12} \mathrm{CO}\right)=2 \times 10^{18} \mathrm{~cm}^{-2}$, (bottom left) Region A2 for $N\left({ }^{12} \mathrm{CO}\right)=4 \times 10^{18} \mathrm{~cm}^{-2}$, and (bottom right) Region B for $N\left({ }^{12} \mathrm{CO}\right)=2 \times 10^{18} \mathrm{~cm}^{-2}$. The thick solid lines denote solutions. The entire range of solutions is not shown.

uncertainty to our solutions and the solution column densities may easily be off by a factor of two from the true values. We stress also that our solutions are averages over the beam.

Our RADEX solutions for regions A, A2, and B show a wide range of solutions such as low $(<30 \mathrm{~K})$ temperature but density $>10^{4} \mathrm{~cm}^{-3}$ and also solutions with lower densities but temperature only constrained to be $>30 \mathrm{~K}$. One way this can be interpreted is a two gas component solution with a moderately dense warm $\left(T_{\text {kin }}>30 \mathrm{~K}\right)$ gas component and a dense cold $\left(T_{\text {kin }} \sim 10-30 \mathrm{~K}\right)$ gas component. In Arp 220, Rangwala et al. (2011) have shown that the mid-J to high-J CO transition lines are tracing the warm/hot gas and the low-J CO transition lines are tracing the cold gas $(T \sim 50 \mathrm{~K})$. The mass of the warm gas is $10 \%$ of the cold gas mass in Arp 220 (Rangwala et al. 2011). This result suggests that even our ${ }^{12} \mathrm{CO} J=3-2$ observations may be most sensitive to the dominant colder gas component that our RADEX solutions allow. In order to trace the warm/hot gas component more definitively, we need to observe Arp 299 in higher ${ }^{12} \mathrm{CO}$ transition lines. Pereira-Santaella et al. (2010) have used mid-infrared Spitzer spectra of IC 694 and NGC 3690 to show that there is $5 \times 10^{8} M_{\odot}$ of warm $\left(T_{\text {kin }}>100 \mathrm{~K}\right)$ $\mathrm{H}_{2}$ gas. Since Arp 299 does have a warm/hot gas component up to $T_{\text {kin }} \sim 500 \mathrm{~K}$, we would expect to see strong emission in higher J-level ${ }^{12} \mathrm{CO}$ lines. The Herschel Fourier Transform Spectrometer spectrum for Arp 299 indeed shows a prominent ${ }^{12} \mathrm{CO}$ transition ladder with strong emission up to ${ }^{12} \mathrm{CO} J=$ 13-12 for regions A and B (M. R. P. Schirm 2011, private communication).

Recently, Papadopoulos et al. (2012) have performed large velocity gradient (LVG) modeling of a single gas phase of Arp 299 using single dish CO transition lines. Papadopoulos et al. (2012) used global ${ }^{12} \mathrm{CO}((J=3-2) /(J=1-0))$, $((J=4-3) /(J=1-0))$ and $\left({ }^{12} \mathrm{CO} /{ }^{13} \mathrm{CO}\right) J=1-0$ line ratios and found a best fit of $T_{\text {kin }}=30 \mathrm{~K}$ and $n\left(\mathrm{H}_{2}\right)=10^{4} \mathrm{~cm}^{-3}$, well 
Table 6

Gas Mass and Depletion Times

\begin{tabular}{|c|c|c|c|c|c|}
\hline Region & $\begin{array}{c}\dot{M}_{\mathrm{SFR}} \\
\left(M_{\odot} \mathrm{yr}^{-1}\right)\end{array}$ & $\begin{array}{c}{ }^{12} \mathrm{CO} J=2-1 \text { Flux } \\
\left(\mathrm{Jy} \mathrm{km} \mathrm{s}^{-1}\right)\end{array}$ & $\begin{array}{c}L_{\mathrm{CO}(2-1)} \mathrm{b}, \mathrm{c} \\
\left(10^{9} \mathrm{~K} \mathrm{~km} \mathrm{~s}^{-1} \mathrm{pc}^{2}\right)\end{array}$ & $\begin{array}{c}M\left(\mathrm{H}_{2}\right)^{\mathrm{c}} \\
\left(10^{8} M_{\odot}\right)\end{array}$ & $\begin{array}{l}t_{\mathrm{depl}}^{\mathrm{c}} \\
(\mathrm{Myr})\end{array}$ \\
\hline $\bar{A}$ & 38.5 & $920 \pm 100$ & $1.2 \pm 0.2$ & $7 \pm 1$ & $25 \pm 5$ \\
\hline $\mathrm{A} 2$ & $<7.7^{\mathrm{d}}$ & $320 \pm 20$ & $0.4 \pm 0.1$ & $2.3 \pm 0.5$ & $>41$ \\
\hline B & 21 & $390 \pm 40$ & $0.5 \pm 0.1$ & $3.0 \pm 0.6$ & $20 \pm 4$ \\
\hline $\mathrm{C}=\mathrm{C} 1+\mathrm{C} 2$ & 10 & $510 \pm 30$ & $0.7 \pm 0.1$ & $4.0 \pm 0.7$ & $50 \pm 10$ \\
\hline Total & 77 & $2380 \pm 20$ & $3.1 \pm 0.6$ & $18 \pm 4$ & $32 \pm 6$ \\
\hline
\end{tabular}

Notes.

${ }^{\text {a }}$ Measurement uncertainty only; calibration uncertainty is $20 \%$.

${ }^{\text {b }}$ Using $D_{\mathrm{L}}=46 \mathrm{Mpc}$.

c Uncertainty is calculated using the calibration uncertainty.

${ }^{\mathrm{d}} \dot{M}_{\text {SFR }}$ calculated assuming the region contains $10 \%$ the total infrared luminosity of Arp 299 .

within our RADEX solutions. Other solutions were shown to be possible with higher temperatures and low densities, similar to our own RADEX solutions for regions A, A2, and B.

\subsection{Arp 299 in Context: Comparison to M33}

LVG modeling has previously been performed on a sample of seven individual giant molecular clouds (GMCs) in the normal spiral galaxy M33 (Wilson et al. 1997). The average of six clouds resulted in a solution of $T_{\text {kin }}=10-20 \mathrm{~K}, n\left(\mathrm{H}_{2}\right)=4-30 \times$ $10^{3} \mathrm{~cm}^{-3}$, and $N\left({ }^{12} \mathrm{CO}\right)=3-6 \times 10^{17} \mathrm{~cm}^{-2}$. This average solution resembles our RADEX solution for region $\mathrm{C} 1$ except for the beam-averaged column density which is significantly lower. Since the sizes of the clouds are known (Wilson \& Scoville 1990) we can determine the average column density over the cloud itself using the filling factor of the cloud within the beam, $f=D_{\text {cloud }}^{2} / D_{\text {beam }}^{2}$, where $D_{\text {cloud }}$ is the diameter of the cloud and $D_{\text {beam }}$ is the physical diameter of the beam $(85 \mathrm{pc})$. Using the equation in Wilson \& Scoville (1990),

$$
V_{\mathrm{FWHM}}=1.2 D_{\mathrm{pc}}^{0.5},
$$

where $V_{\mathrm{FWHM}}$ is the line width FWHM of the CO line in $\mathrm{km} \mathrm{s}^{-1}$ and $D_{\mathrm{pc}}$ is the diameter of the cloud in pc and using the average $V_{\text {FWHM }}$ for the six clouds given in Wilson et al. (1997) $\left(=9.3 \mathrm{~km} \mathrm{~s}^{-1}\right)$, we get an average $D_{\text {cloud }}$ of $60 \mathrm{pc}$. This gives us a filling factor of $f=0.5$ and a cloud-averaged column density of $N\left({ }^{12} \mathrm{CO}\right)=6-12 \times 10^{17} \mathrm{~cm}^{-2}$ for the M33 clouds. Comparing the cloud-averaged column density value to the ${ }^{12} \mathrm{CO}$ column density of region $\mathrm{C}$, we see that the sub-region $\mathrm{C} 1$ and even $\mathrm{C} 2$ have at least 2-3 times the average column density of the clouds in M33. The striking thing is that this column density is achieved not just in an individual GMC but over a $\sim 1 \mathrm{kpc}$ diameter region. This analysis implies that if region $\mathrm{C}(=\mathrm{C} 1+\mathrm{C} 2)$ is made up of normal GMCs such as those in M33, the beam is filled with clouds spaced very close together such that the entire $\sim 1 \mathrm{kpc}$ region has a structure similar to a single GMC and with at least 2-3 clouds along each line of sight. This unusual structure may help explain the short gas depletion time and high star formation rate observed in region C in particular and Arp 299 as a whole (see Section 4).

We can also calculate the average volume density of region $\mathrm{C}$ assuming a spheroidal geometry. The average radius in the plane of the sky of region $\mathrm{C}$ is $R \sim 350 \mathrm{pc}$. However, a diameter of $\sim 700 \mathrm{pc}$ along the line of sight would be much greater than the typical disk scale height. From the column density comparison, we might expect to encounter a maximum of three GMCs of about $60 \mathrm{pc}$ in diameter. If the space in between the clouds is negligible then the thickness of region $\mathrm{C}$ is about 200 pc. Using the molecular gas mass (Section 4 and Table 6) and $\left\langle n\left(\mathrm{H}_{2}\right)\right\rangle=3 M_{\mathrm{H}_{2}} / 4 \pi m_{\mathrm{H}_{2}} R^{3}$ we derive an average volume density of $130 \mathrm{~cm}^{-3}$. We can compare this average volume density to the average value of the six clouds in M33. Using the equation given in Wilson \& Scoville (1990)

$$
\left\langle n\left(\mathrm{H}_{2}\right)\right\rangle=200\left(D_{\mathrm{pc}} / 20 \mathrm{pc}\right)^{-1}
$$

and the average cloud diameter, we get an average volume density of about $70 \mathrm{~cm}^{-3}$ for the clouds in M33. This comparison implies that region $\mathrm{C}$ is much denser than a 60 pc cloud in M33, even though it covers a much larger spatial scale.

It has been suggested previously that U/LIRGs have kiloparsec scale medium that is like a GMC as a whole (e.g., Downes \& Solomon 1998). An alternative possibility that could reproduce the observed large column density is that U/LIRGs contain a medium where the mean density of individual clouds and clumps is much higher than typical GMCs (perhaps similar to the circumnuclear disk in the Galactic center i.e., Christopher et al. 2005). However, this second situation would result in a radiative transfer solution volume density $\left(n_{\text {RADEX }}\right)$ much greater than that of the clouds in M33 $\left(n_{\mathrm{LVG}}(\mathrm{M} 33)\right)$. For region C, we see that $n_{\mathrm{RADEX}} \sim n_{\mathrm{LVG}}(\mathrm{M} 33)$ ruling out the alternative suggestion. Imanishi \& Nakanishi (2006) have found that interferometric $\mathrm{HCN} / \mathrm{HCO}+J=1-0$ line ratios of each region are less than 1 likely indicating that there is a lack of very dense gas, which also supports our results. This is the first direct evidence from a radiative transfer analysis using spatially resolved data of a kiloparsec scale structure having the properties of a GMC in a U/LIRG.

\subsection{CO-to- $\mathrm{H}_{2}$ Conversion Factor in Region Cof Arp 299}

Since region $\mathrm{C} 1$ has a better constrained radiative transfer solution, we can examine both our assumed ${ }^{12} \mathrm{CO}$ abundance $\left(x_{\mathrm{co}}=3 \times 10^{-4}\right)$ and also the value of the CO-to- $\mathrm{H}_{2}$ conversion factor. We do this by comparing the RADEX solution's ${ }^{12} \mathrm{CO}$ column density to the average $\mathrm{H}_{2}$ column density within the beam derived from the ${ }^{12} \mathrm{CO}$ intensity. The $\mathrm{CO}$ luminosity of each region can be calculated using the equation

$$
\begin{aligned}
\frac{L^{\prime}}{\mathrm{Kkm} \mathrm{s}^{-1} \mathrm{pc}^{2}}= & 3.2546 \times 10^{7}\left(\frac{S_{\mathrm{CO}}}{\mathrm{Jy} \mathrm{km} \mathrm{s}^{-1}}\right) \\
& \times\left(\frac{D_{L}}{\mathrm{Mpc}}\right)^{2}\left(\frac{v_{0}}{\mathrm{GHz}}\right)^{-2}(1+z)^{-1},
\end{aligned}
$$

where $S_{\mathrm{CO}}$ is the flux of the CO line in $\mathrm{Jy} \mathrm{km} \mathrm{s}^{-1}, D_{\mathrm{L}}$ is the luminosity distance in $\mathrm{Mpc}, v_{\mathrm{o}}$ is the rest frequency of the 
$\mathrm{CO}$ line in $\mathrm{GHz}$, and $z$ is the redshift (Paper I). Since the ${ }^{12} \mathrm{CO} J=1-0$ map does not include short spacings and we do not know how much flux is missing in each region, we use the short spacing corrected ${ }^{12} \mathrm{CO} J=2-1$ map to measure the gas masses of each region. We use an average ratio value of ${ }^{12} \mathrm{CO}((J=2-1) /(J=1-0))=1.4$ (Table 4$)$ to estimate the gas mass, which is then given by

$$
M\left(\mathrm{H}_{2}\right)=0.57 L_{\mathrm{CO}(2-1)}^{\prime},
$$

where $L_{\mathrm{CO}(2-1)}^{\prime}$ is given by Equation (3) and we assume $\alpha_{\text {co }}=0.8 M_{\odot}\left(\mathrm{K} \mathrm{km} \mathrm{s}^{-1} \mathrm{pc}^{2}\right)^{-1}$ for the ${ }^{12} \mathrm{CO} J=1-0$ line (Downes \& Solomon 1998).

Using the short spacing corrected ${ }^{12} \mathrm{CO} J=2-1$ map at the degraded resolution, the peak intensity of region $\mathrm{C} 1$ is $170 \mathrm{Jy}$ beam $^{-1} \mathrm{~km} \mathrm{~s}^{-1}$ or $2.2 \times 10^{8} \mathrm{~K} \mathrm{~km} \mathrm{~s}^{-1} \mathrm{pc}^{2}$ which corresponds to a mass of $M_{\mathrm{H}_{2}}=1.3 \times 10^{8} M_{\odot}$ using Equation (4). With a beam size of 3". $6 \times 2$ ". 4 this corresponds to an average $\mathrm{H}_{2}$ column density of $1.7 \times 10^{22} \mathrm{~cm}^{-2}$. Using the ${ }^{12} \mathrm{CO}$ column density obtained from the RADEX solution, we get a ${ }^{12} \mathrm{CO}$ abundance of $x_{\text {co }}=N\left({ }^{12} \mathrm{CO}\right) / N\left(\mathrm{H}_{2}\right)=0.6-2.4 \times$ $10^{-4}$. This is 1.3-5 times lower than the abundance ratio of $3 \times 10^{-4}$ that is typically assumed (Ward et al. 2003). This difference may be interpreted in two ways. One possibility is that the abundance of ${ }^{12} \mathrm{CO}$ to $\mathrm{H}_{2}$ is in fact lower in this region of Arp 299 than the average Galactic value. Relaño et al. (2007) have reported a metallicity that is near-solar for Arp 299 which would suggest that the abundance of ${ }^{12} \mathrm{CO}$ to $\mathrm{H}_{2}$ should not differ greatly from the value that is typically used. If we use the updated work by Dickman (1978) for "dark clouds" reported in Magnani et al. (1988), $N\left(\mathrm{H}_{2}\right)=(4 \pm 2) \times 10^{5} N\left({ }^{13} \mathrm{CO}\right)$ and our assumed $\left[{ }^{12} \mathrm{CO}\right] /\left[{ }^{13} \mathrm{CO}\right]$ of 50 , we get an abundance of $x_{12 \mathrm{CO}}=$ $(1.3 \pm 0.7) \times 10^{-4}$. This value agrees well with our analysis. A second possibility is that the ${ }^{12} \mathrm{CO}$ abundance in Arp 299 is the same as in the Galaxy but the value of the CO-to- $\mathrm{H}_{2}$ conversion factor, $\alpha_{\mathrm{co}}$, is smaller than $0.8 M_{\odot}\left(\mathrm{K} \mathrm{km} \mathrm{s}^{-1} \mathrm{pc}^{2}\right)^{-1}$. If we use the peak intensity value of the ${ }^{12} \mathrm{CO} J=2-1$ map and the mass within the beam derived from the column density solution of region $\mathrm{C} 1$ we get

$$
\alpha_{\mathrm{CO}(1-0)}=0.4 \pm 0.3\left(\frac{3 \times 10^{-4}}{x_{\mathrm{co}}}\right) M_{\odot}\left(\mathrm{K} \mathrm{km} \mathrm{s}^{-1} \mathrm{pc}^{2}\right)^{-1}
$$

To get $\alpha_{\mathrm{CO}}$ to agree with Downes \& Solomon (1998), the $x_{\text {co }}$ abundance factor must be $\sim 1.5 \times 10^{-4}$, lower than our assumed value by a factor of two. There is no way of telling which scenario is right and it may well be a combination of the two. Papadopoulos et al. (2012) have also found a CO-to$\mathrm{H}_{2}$ factor over their global LVG solutions to be $0.35-0.42 M_{\odot}$ $\left(\mathrm{K} \mathrm{km} \mathrm{s}^{-1} \mathrm{pc}^{2}\right)^{-1}$. This range of values agrees well with the value we estimated for region $\mathrm{C} 1$ using the RADEX solutions. Note that our result is independent of the method used by Downes \& Solomon (1998).

We can attempt to place a second limit on the CO-to$\mathrm{H}_{2}$ conversion factor using the dynamical mass of region $\mathrm{C}$ $(=\mathrm{C} 1+\mathrm{C} 2)$. The dynamical mass (Table 3$)$ can be estimated using the equation from Wilson \& Scoville (1990):

$$
M_{\text {dyn }}=99 \Delta V_{\mathrm{FWHM}}^{2} D(\mathrm{pc}) M_{\odot},
$$

where $\Delta V_{\mathrm{FWHM}}$ is the CO line FWHM in $\mathrm{km} \mathrm{s}^{-1}$ and $D(\mathrm{pc})$ is the diameter of the region in pc. Note that Equation (6) is valid only for regions that are gravitationally bound. If we assume that the dynamical mass is entirely due to molecular gas, then $\alpha_{\mathrm{CO}}=M_{\mathrm{dyn}} / L_{\mathrm{CO}}^{\prime}$. Using the total flux for region $\mathrm{C}$ from the short spacing corrected ${ }^{12} \mathrm{CO} J=2-1$ map and a ${ }^{12} \mathrm{CO}$ $2-1 / 1-0$ average ratio of 1.4 (Table 4 ), we find that $\alpha_{\mathrm{CO}}=$ $1.4 M_{\odot}\left(\mathrm{K} \mathrm{km} \mathrm{s}^{-1} \mathrm{pc}^{2}\right)^{-1}$. However, the dynamical mass is likely greater than the true molecular gas mass as stars may contribute some mass and the region as a whole may not be gravitationally bound. Thus, this value of $\alpha_{\mathrm{CO}}$ is strictly speaking an upper limit.

\section{STAR FORMATION RATES AND DEPLETION TIMES}

We estimate the far-infrared luminosity of each region using $L_{\mathrm{FIR}}=5.5 \times 10^{11} L_{\odot}($ Sanders et al. 2003) for Arp 299 and the fractions of the total luminosity for each region from Alonso-Herrero et al. (2000) based on ground-based midinfrared observations. From the relative fluxes at 10, 12, 20, and $30 \mu \mathrm{m}$ through a $6^{\prime \prime}$ diameter, Alonso-Herrero et al. (2000) estimated that region $\mathrm{A}$ has about $50 \%$, region $\mathrm{B}$ has about $27 \%$, and region $\mathrm{C}$ has about $13 \%$ of the total infrared luminosity. The final $10 \%$ is believed to be associated with $\mathrm{H}$ II regions. The star formation rate can be estimated from the infrared luminosity $(8-1000 \mu \mathrm{m})$ using the equation from Kennicutt (1998), $\dot{M}_{\mathrm{SFR}}\left(M_{\odot} \mathrm{yr}^{-1}\right)=4.5 \times 10^{-44} L_{\mathrm{IR}}\left(\mathrm{erg} \mathrm{s}^{-1}\right)$. Kennicutt (1998) used a Salpeter (Salpeter 1955) initial mass function (IMF) to estimate the star formation rate; a double power law IMF with a slope of -1.3 in the range of $0.1-0.5 M_{\odot}$ and a slope of -2.3 in the range of $0.5-120 M_{\odot}$ is more commonly used (i.e., Calzetti et al. 2007). The star formation rate is 1.59 times lower with the double power law IMF than with the Salpeter IMF. To minimize any contribution to the infrared luminosity from any AGNs present in Arp 299, we adopt the method of Genzel et al. (2010), who scale the far-infrared luminosity by a factor of 1.3 to estimate the total infrared $(8-1000 \mu \mathrm{m})$ luminosity associated with star formation. This method avoids using the directly measured mid-infrared luminosity that is more likely to be contaminated by an AGN. The correction factor of 1.3 is an average $L_{\mathrm{IR}} / L_{\mathrm{FIR}}$ in nearby star-forming galaxies (GraciáCarpio et al. 2008). With these changes, the star formation rate becomes

$$
\dot{M}_{\mathrm{SFR}}\left(M_{\odot} \mathrm{yr}^{-1}\right)=1.4 \times 10^{-10} L_{\mathrm{FIR}}\left(L_{\odot}\right) .
$$

Table 6 summarizes the derived properties of Arp 299.

Using the total molecular gas mass and star formation rates of each region, we can estimate the amount of time it will take for all the molecular gas to be depleted by star formation using

$$
t_{\mathrm{depl}}=\frac{1.36 M\left(\mathrm{H}_{2}\right)}{\dot{M}_{\mathrm{SFR}}},
$$

where the factor 1.36 is to account for the helium gas. The depletion times of each region (Table 6) are very short compared to normal spiral galaxies (Leroy et al. 2008). The timescales are more similar to the lifetime of GMCs in the Large Magellanic Cloud (20-30 Myr; Kawamura et al., 2009). Daddi et al. (2010) and Genzel et al. (2010) studied the star formation laws in disk galaxies and starbursts at low and high redshifts. They provide evidence that disks and starbursts occupy distinct regions in the molecular gas mass $\left(M_{\mathrm{H}_{2}}\right)$ versus star formation rate (or $L_{\mathrm{IR}}$ ) plane. Starbursts are seen to have 10 times higher $L_{\mathrm{IR}}$ at fixed $M_{\mathrm{H}_{2}}$. U/LIRGs have depletion times on the order of $10^{7}$ years while spiral galaxies have depletion times in the range of 0.6-2.4 Gyr (Daddi et al. 2010).

To increase the gas depletion times in Arp 299 to values similar to normal spiral galaxies, either the molecular gas mass is 
underestimated or the star formation rate is overestimated. The molecular gas mass must be increased by two orders of magnitude to achieve similar depletion times to those seen in normal spiral galaxies. However, even if we take the dynamical masses to be entirely molecular gas, we do not get close to increasing the gas mass by this amount. If the far-infrared luminosity is heavily contaminated by an AGN then the star formation rates could be overestimated. If we take the extreme case that an AGN contributes $80 \%$ to the total infrared luminosity, the largest contribution found for any U/LIRG by Genzel et al. (1998), the depletion timescales in the two nuclei would only be increased by a factor of five. In either case, taking the molecular gas mass or the star formation rates to the extreme limits does not give us depletion timescales similar to those of normal spiral galaxies.

The global gas depletion time of Arp 299 suggests that all of the molecular gas will be used up in star formation in about half of the merger time ( 60 Myr; Hibbard \& Yun 1999). If the molecular gas is not replenished over time, Arp 299 may not reach the ULIRG phase. However, Hibbard \& Yun (1999) have detected a tidal tail containing $3.3 \times 10^{9} M_{\odot}$ of $\mathrm{H}$. If the gas inflow rate is sufficiently fast then there could be replenishment of molecular gas for further star formation.

\section{CONCLUSIONS}

We have analyzed the interferometric $\mathrm{CO}$ observations of the local LIRG Arp 299 first published in Wilson et al. (2008). CO line ratios have been used to constrain the physical conditions of each region of Arp 299 using the radiative transfer code RADEX. Our RADEX solutions for regions A, A2, and B show two situations, solutions with low $(<30 \mathrm{~K})$ temperature but density only constrained to be $>10^{4} \mathrm{~cm}^{-3}$ and also solutions with lower densities but temperature only constrained to be $>30 \mathrm{~K}$. This analysis allows for the possibility of a two component gas, but higher J-level ${ }^{12} \mathrm{CO}$ transition lines are required to trace the warm gas conclusively.

The sub-region $\mathrm{C} 1$ corresponding to the peak intensity overlap region of Arp 299 shows a better-constrained RADEX solution with $T_{\text {kin }} \sim 10-50 \mathrm{~K}, n\left(\mathrm{H}_{2}\right) \sim 1-30 \times 10^{3} \mathrm{~cm}^{-3}$, and $N\left({ }^{12} \mathrm{CO}\right) \sim 1-4 \times 10^{18} \mathrm{~cm}^{-2}$. This solution is comparable to that of GMCs in the normal spiral galaxy M33. The column densities of the clouds of M33 are at least 2-3 times lower than the column density for region $\mathrm{C}(=\mathrm{C} 1+\mathrm{C} 2)$ which may indicate that clouds are spaced close together in region $\mathrm{C}$. We estimate the CO-to- $\mathrm{H}_{2}$ factor, $\alpha_{\text {co }}$ to be $0.4 \pm 0.3\left(3 \times 10^{-4} / x_{\mathrm{co}}\right) M_{\odot}$ $\left(\mathrm{K} \mathrm{km} \mathrm{s}^{-1} \mathrm{pc}^{2}\right)^{-1}$ for region $\mathrm{C} 1$, which agrees well with Downes \& Solomon (1998) for more compact and luminous ULIRGs. Using the dynamical mass of region $\mathrm{C}(=\mathrm{C} 1+\mathrm{C} 2)$, an upper limit to $\alpha_{\mathrm{CO}}$ was determined to be $1.4 M_{\odot}\left(\mathrm{K} \mathrm{km} \mathrm{s}^{-1} \mathrm{pc}^{2}\right)^{-1}$

We have used the $\mathrm{CO}$ observations to calculate the gas and dynamical masses of each region and infrared data to derive the star formation rate of each region. We find that the gas depletion times of each region (20-50 Myr) are about two orders of magnitude lower than those for normal spiral galaxies and resemble the 30 Myr depletion timescales of GMCs. These short depletion timescales may be explained by a higher concentration of dense gas than in normal spiral galaxies. If the molecular gas is not replenished over time, Arp 299 will run out of fuel for star formation in about half the time it will take for the nuclei to finally merge.
The Submillimeter Array is a joint project between the Smithsonian Astrophysical Observatory and the Academia Sinica Institute of Astronomy and Astrophysics and is funded by the Smithsonian Institution and the Academia Sinica. The James Clerk Maxwell Telescope is operated by The Joint Astronomy Centre on behalf of the Particle Physics and Astronomy Research Council of the United Kingdom, the Netherlands Organisation for Scientific Research, and the National Research Council of Canada. We thank the anonymous referee for a very useful referee report and S. Aalto for giving us the OVRO CO $J=1-0$ map. C.D.W. acknowledges support by the Natural Science and Engineering Research Council of Canada (NSERC).

\section{REFERENCES}

Aalto, S., Booth, R. S., Black, J. H., \& Johansson, L. E. B. 1995, A\&A, 300,369

Aalto, S., Radford, S. J. E., Scoville, N. Z., \& Sargent, A. I. 1997, ApJ, 475, L107

Alonso-Herrero, A., Rieke, G. H., Rieke, M. J., \& Scoville, N. Z. 2000, ApJ, 532,845

Armus, L., Heckman, T., \& Miley, G. 1987, AJ, 94, 831

Barnes, J. E., \& Hernquist, L. E. 1991, ApJ, 370, L65

Calzetti, D., Kennicutt, R. C., Engelbracht, C. W., et al. 2007, ApJ, 666, 870

Casoli, F., Willaime, M.-C., Viallefond, F., \& Gerin, M. 1999, A\&A, 346, 663

Christopher, M. H., Scoville, N. Z., Stolovy, S. R., \& Yun, M. S. 2005, ApJ, 622,346

Currie, M. J., Draper, P. W., Berry, D. S., et al. 2008, in ASP Conf. Ser. 394, Astronomical Data Analysis Software and Systems XVII, ed. R. W. Argyle, P. S. Bunclark, \& J. R. Lewis (San Francisco, CA: ASP), 650

Daddi, E., Elbaz, D., Walter, F., et al. 2010, ApJ, 714, L118

Dickman, R. L. 1978, ApJS, 37, 407

Downes, D., \& Solomon, P. M. 1998, ApJ, 507, 615

Gehrz, R. D., Sramek, R. A., \& Weedman, D. W. 1983, ApJ, 267, 551

Genzel, R., Lutz, D., Sturm, E., et al. 1998, ApJ, 498, 579

Genzel, R., Tacconi, L. J., Gracia-Carpio, J., et al. 2010, MNRAS, 407, 2091

Graciá-Carpio, J., García-Burillo, S., Planesas, P., \& Colina, L. 2006, ApJ, 640, L135

Graciá-Carpio, J., García-Burillo, S., Planesas, P., Fuente, A., \& Usero, A. 2008, A\&A, 479, 703

Hibbard, J. E., \& Yun, M. S. 1999, AJ, 118, 162

Ho, P. T. P., Moran, J. M., \& Lo, K. Y. 2004, ApJ, 616, L1

Imanishi, M., \& Nakanishi, K. 2006, PASJ, 58, 813

Iono, D., Wilson, C. D., Yun, M. S., et al. 2009, ApJ, 695, 1537

Kawamura, A., Mizuno, Y., Minamidani, T., et al. 2009, ApJS, 184, 1

Kennicutt, R. C., Jr. 1998, ARA\&A, 36, 189

Leroy, A. K., Walter, F., Brinks, E., et al. 2008, AJ, 136, 2782

Magnani, L., Blitz, L., \& Wouterloot, J. G. A. 1988, ApJ, 326, 909

Menéndez-Delmestre, K., Blain, A. W., Smail, I., et al. 2009, ApJ, 699, 667

Murphy, T. W., Jr., Armus, L., Matthews, K., et al. 1996, AJ, 111, 1025

Papadopoulos, P. P., van der Werf, P., Xilouris, E., Isaak, K. G., \& Gao, Y. 2012, ApJ, 751, 10

Pereira-Santaella, M., Alonso-Herrero, A., Rieke, G. H., et al. 2010, ApJS, 188, 447

Rangwala, N., Maloney, P. R., Glenn, J., et al. 2011, ApJ, 743, 94

Relaño, M., Lisenfeld, U., Pérez-González, P. G., Vílchez, J. M., \& Battaner, E. 2007, ApJ, 667, L141

Salpeter, E. E. 1955, ApJ, 121, 161

Sanders, D. B., Mazzarella, J. M., Kim, D.-C., Surace, J. A., \& Soifer, B. T. 2003, AJ, 126, 1607

Sanders, D. B., \& Mirabel, I. F. 1996, ARA\&A, 34, 749

Sanders, D. B., Scoville, N. Z., Young, J. S., et al. 1986, ApJ, 305, L45

Sanders, D. B., Soifer, B. T., Elias, J. H., et al. 1988, ApJ, 325, 74

Sargent, A., \& Scoville, N. 1991, ApJ, 366, L1

van der Tak, F. F. S., Black, J. H., Schöier, F. L., Jansen, D. J., \& van Dishoeck, E. F. 2007, A\&A, 468, 627

Ward, J. S., Zmuidzinas, J., Harris, A. I., \& Isaak, K. G. 2003, ApJ, 587, 171

Wilson, C. D., Petitpas, G. R., Iono, D., et al. 2008, ApJS, 178, 189

Wilson, C. D., \& Scoville, N. 1990, ApJ, 363, 435

Wilson, C. D., Walker, C. E., \& Thornley, M. D. 1997, ApJ, 483, 210 